\title{
Food environment as a healthy lifestyle factor
}

\author{
Nikolay Belyaev*, Ludmila Donskova, and Boisjoni Tohiriyon \\ Urals State University of Economics, 8 March / Narodnaya Volya Str., 62/45, 620144 Ekaterinburg, \\ Russia
}

\begin{abstract}
The authors consider food products in the context of ensuring a healthy lifestyle. Arguing with the results of studies of foreign and domestic scientists the relationship between food and a healthy lifestyle, as well as well-being, and developing the theory of a healthy food environment, the main parameters of the formation of a healthy food environment have been established: first of all the production and consumption of high-quality and safe food products. The directions of the market development for functional food products at the federal level are determined, vectors of research in relation to social toxicants are indicated. The authors believe that the main provisions of this study can serve to build a holistic, balanced and effective theory of a healthy food environment development, as a factor ensuring a healthy lifestyle and wellbeing of people at any age, and can also be useful for the business community in terms of possible investments, and for consumers, as key subjects of the implemented concept of sustainable development.
\end{abstract}

\section{Introduction}

It is estimated that the world's population could surpass 9.5 billion in 2050 (United Nations, 2014), an increase of $32 \%$ of the current population, and therefore food production alone must grow by $60 \%$ to keep up with this growth, mainly urban population. Therefore, sustainable growth is one of the millennium goals, which must be achieved for the survival of the planet [1].

The FAO / WHO documents on sustainable development highlight, that ensuring healthy lifestyles and promoting well-being for all people at all ages are viewed as constituents of sustainable development, being essential for building a prosperous society.

A healthy lifestyle and a good health begin with nutrition. Without regular consumption of qualitative and safe food, a person cannot live, study, fight disease, and maintain emotional and physical health.

Many countries, supporting the sustainable development concept, have developed their own nationally adapted policies and strategies for promoting healthy lifestyles and wellbeing of population at any age. For example, in October 2016, to improve the Canadian nutritional landscape, Health Canada launched the Healthy Eating Strategy, which included a number of initiatives in terms of limiting the marketing of unhealthy foodstuffs for children, reducing sodium in food, introducing labeling on packaging, etc. [2]. The Strategy

*Corresponding author: cafedra@list.ru 
for the formation of a healthy lifestyle of the population for the period up to 2025 in the Russian Federation was approved.

As underlined by J. A. Nitzke et al. [1], the discussion of the relationship between nutrition and health reflects the global consciousness that food does not play only a nutritional role, but has a deep relationship with almost every aspect (nutritional, sensory, social, cultural, ecological, anthropological, etc.) in our daily life. The idea "Food health" [1] was extended by foreign scientists in order to discuss issues related to food safety, however, in our opinion, it is much broader and covers various aspects of production, logistics, sale and consumption of food products, thereby forming a healthy food environment.

An unhealthy food environment contributes to increasing obesity and nutrition-related diseases, as evidenced by the results of numerous studies conducted by the foreign and domestic scientists in different periods.

Non-communicable diseases (NCDs), which include cardiovascular, chronic bronchopulmonary diseases, malignant tumors, diabetes mellitus and others, are considered as a threat of the 21 st century and they are called the diseases of civilization, resulting in more than $60 \%$ of deaths in the Russian Federation; every year 1.29 million people die from NCDs in Russia.

At the heart of the non-communicable diseases development are risk factors associated with an unhealthy lifestyle, embracing smoking, alcohol abuse, unhealthy diet, and lack of physical activity. High blood pressure, hyperglycemia, high cholesterol are the consequences of the unhealthy food environment.

The analysis of scientific publications proved that the food products issue is covered by foreign and domestic scientists in various aspects from global problems of our time: food security, including during a pandemic, food safety, food losses, fraud and falsification in production and trade, innovative technologies in food science related to certain aspects of food chemistry, consumption of specific products, marketing and logistics. However, in the context of ensuring a healthy lifestyle, information requires systematization and adaptation to the stated goals. The analysis of scientific data shows that this topic is multifaceted, possessing a general theoretical character; in applied issues of a debatable nature, requiring research and analysis based on a systematic and integrated approach, predetermining the choice of the research trend.

In this connection, the purpose of these studies, the main provisions of which are reflected in the article, was to study the theoretical and practical aspects of a healthy food environment formation as a healthy lifestyle provision factor.

\section{Methods}

For implementing a set goal, bibliographic, systemic, statistical, logical, methods of analysis and synthesis were used as theoretical ones, as well as methods of publication research and expert assessments were used for analyzing applied issues.

\section{Results and Discussion}

First of all, a healthy food environment is determined by a sufficient amount of food products.

The world has enough food stocks for feeding the world's 7.8 billion people. Nevertheless, more than 820 million people are hungry today, about 144 million, or more than one-fifth of children under the age of five are globally stunted, 6.9 percent (47.0 million) are emaciated and 5.6 percent (38.3 million) suffer from overweight. Food systems 
are working intermittently, and the COVID-19 pandemic, aggravating the situation, could add to the total number of starving people in the world from 83 to 132 millions [3]. These issues related to starvation are the subject of SDG 2 - Zero Hunger and are not investigated in this article.

The food environment is formed by a variety of food products that people use for nutrition. The results of many studies conducted by foreign and domestic scientists indicate that many products are useful for human health and well-being, and among them there are those that are directly related to the reduction of chronic diseases. At the same time, the formation of a healthy food environment is linked inseparably to the issues of food quality and safety. For example, G. Kaptan et al. facilitate that seafood, in particular fish, are important suppliers of omega-3 fatty acids, and their consumption can help prevent certain diseases such as cardiovascular one.

However, fish is also associated with the environmental pollutants such as methylmercury and organochlorine compounds, which, especially methylmercury, can have adverse effects on fetal development, while organochlorine compounds can adversely affect the liver, kidneys and the central nervous system [4]. Thus, the health of food products is provided, above all, by their safety. Among the most relevant risks are allergenic foods, aluminum additives, water footprint in food production, food fraud, food supply chain management and their verification [5]. In most countries, consumers' perception of certain risks is associated with foodstuffs, including genetically modified organisms (GMOs), the presence of pesticides and food additives, etc. Food products that are considered more natural, such as organic and non-GMO foodstuffs, are generally perceived as less risky / healthier [2]. Among the most serious problems connected with food safety, the scientists pointed out the following points: transparency of the entire food production chain, harmonization of international norms and standards, the ability to grow and cope with emerging risks.

Food fraud has been and is likely to remain a serious problem when we think of "food health", in some cases there are only economic problems that threaten consumer rights or product authenticity, but very often this can lead to serious health problems and even death. In general, the world community has formed the main statements in the field of food safety in the form of international and national documents, which represent the basic theoretical and practical basis for the development of this area at the present time.

There is an increasing evidence in the nutritional literature and authoritative reports about the concept of ultra-processed foods (UPF), recognized as an unhealthy diet. The evidence proves that high consumption of ultra-processed foods (UPFs) has a positive connection between UPFs consumption and the risk of multiple health effects, determined by an increase in non- communicable diseases, overweight and obesity [6, 7].

However, when we talk about a healthy food environment, in addition to negative aspects such as counterfeiting, infection or the presence of pathogens, it is worth mentioning the importance of using new technologies to increase the "functionality" of foodstuff and other health benefits, as well as to improve their quality, especially consumed in underdeveloped or developing countries. The functional foodstuffs and nutritional supplements containing fruit extracts and herbal products attract more attention.

In the early 1980s, the Japanese scientific community defined the concept of "functional food". The scientists have proposed to consider food in three dimensions: ensuring the supply of essential substances to the body, providing satisfaction in sensory characteristics, and, finally, the tertiary function of the food, aimed at improving health and reducing the risk of disease [8]. The new system was focused on developing the production of food products and solving serious health problems. And today the Japanese government recognize functional nutrition, as evidenced by $39.2 \%$ of the global market of functional foods [9], defining it as an alternative to drug therapy Food for Specific Health Use 
(FOSHU), highlighting several categories of functional foodstuffs [10]. The range of functional products in Russia began to develop actively after the adoption of the "Concept of state policy in the field of healthy nutrition of the population of Russia." Unfortunately, the production of domestic functional products has not reached the federal scale: they are produced mainly at separate enterprises that work with research centers engaged in developing functional products [9]. In the process of creating functional products, there are problems, determined by new scientific approaches to solve them. From the food industry perspective, the development of functional foods often requires an increased level of complexity and the food industry monitoring. This is due to the fact that new raw materials can be used, including those obtained by implementing biotechnology, new thermal and non-thermal technologies, new safety issues, and integration within the framework of the entire food chain needs to be revised, especially to ensure conservation and / or expansion of products functionality [11].

As consumers demand more and more health-related foodstuffs, it would be very complicated to enrich all food products with functional ingredients that they do not naturally possess, such as lipophilic BACs, probiotics, proteins / peptides, etc. Currently, great potential for food innovation can be achieved with the help of 3D foodstuffs printing. This is the technology for the production of three-dimensional food products of any shape and size, with preferred palatability and desired nutritional compositions; it could be a promising tool for the inclusion of sensitive and easily degradable biologically active substances and other functional ingredients in functional 3DP foods, which will greatly contribute to producing healthy food [12].

The third direction, considered in the article, is associated with the active development of the production and consumption of food products containing substances that affect the central nervous and cardiovascular systems. The topic "Social toxicants" usually means drugs, alcohol and smoking, and their usage. The healthy lifestyle promotion involves the development and adoption of measures to reduce the consumption and abuse of social toxicants. Excessive alcohol consumption is the leading cause of morbidity and mortality worldwide. The prospect of the development of alcohol-related diseases is shifting from the traditional aspect of direct issue damage caused by alcohol and its metabolites to the involvement of intestinal microbes. The article by A.B. Lisitsyn et al. manifests that alcohol does not only alter the gut environment, but also modulates the composition of the gut microbiota and is involved in the development of alcohol-related diseases. However, the authors state that the research papers about the effects of alcohol beverages on gut microbiota and alcohol-induced trauma is limited [13]. In addition, high consumption of both soft drinks (at least five times a week) and foods with high saturated fat level also contribute to the obesity increase [14].

It is worth noting that the information is presented in a generalized context; it is not systematized and does not take into account those new directions in expanding the range of beverages and tobacco products and other goods that can be viewed as the social toxicants.

\section{Conclusions}

Such trends as replacing traditional natural foods rich in fiber, vitamins, minerals and other biologically active compounds by more industrialized products but inferior in nutritional value, access and preference for convenience stores, changes in activities that led to food consumption outside the home (fast food, energy drinks, foodstuffs with sweeteners, high fat and trans fatty acid isomers) and other reasons have led to the increase in the number of people with such diseases as obesity, diabetes mellitus and others from the list of civilization diseases, and in addition, contributed to creating an unhealthy food environment. 
In the context of promoting a healthy lifestyle in terms of strengthening connection between food and a healthy lifestyle, it is necessary to create a healthy food environment. The authors acknowledge that there will always be different points of view, tools and developments to problem - solving. Undoubtedly, countries must create conditions and tools for ensuring quantitatively and qualitatively adequate production and safe food supply. Joint efforts and increased government support are needed in order to solve crucial problems related to counterfeiting and fraud, new technologies, food additives and food safety issues (alimentary pathogens, impurities, mycotoxins, etc.).

Faced with new trends in healthy nutrition, it became necessary to develop and expand the functional food products market, which will also contribute to forming a healthy food environment and a consumer should be provided with the information about functional food products of any food group that is not even associated with the functional nutrition.

According to the K.S. Petrovsky' point of view, it is impossible to develop one diet acceptable for all healthy people at once, it is necessary to take into account the individual characteristics of a person. However, developing general guidelines, such as those recommended by the $\mathrm{WHO}$, in terms of eating five or more servings of fruits and vegetables, will be a powerful tool against the obesity. In addition, the daily consumption of fruits and vegetables has a preventive effect against a number of chronic diseases, mainly due to their biologically active compounds (BAC) and powerful antioxidant activity. The emphasis on increasing the consumption of fruits and vegetables for consumers, limiting the content of trans-isomers of fatty acids, salt, sugar and other components on the basis of regulatory documents in the production of food products should be the basic principles of a healthy food environment.

Due to a narrow representation of the stated issues in the scientific papers about the classification and assortment, identification and safety assessment of "energy" drinks, "nonalcoholic" beer, drinks using guarana seeds, smoking mixtures, etc., there are a number of questions to deal with. There is an urgent need to collect and organize factual data, draw valuable insights and put them into practice for reducing the potential threats.

\section{Acknowledgements}

The authors would like to thank the interpreter and the editor for their professional approach to work, as well as the colleagues for their helpful suggestions.

\section{References}

1. J. A. Nitzke, F. Cladera, R. Thys, F. T. Da Cruz, S. Martinelli, Food Sci. Technol, Campinas, 36 (2016)

2. D. Vandenbrink, E. Pauzé and M. Potvin Kent, International Journal of Behavioral Nutrition and Physical Activity, 17(3) (2020)

3. FAO, IFAD, UNICEF, WFP and WHO. The State of Food Security and Nutrition in the World 2020. Transforming food systems for affordable healthy diets. Rome, FAO, (2020)

4. G. Kaptan, Arnout R.H. Fischer, L. J. Frewer. Journal of Risk Research (2017)

5. Food and Agriculture Organization - FAO. The state of food insecurity in the world 2015. Rome: FAO, (2015) http:// www.fao.org/hunger/en/

6. L. Elizabeth, P. Machado, M. Zinöcker, P. Baker, M. Lawrence. Nutrients, 12 (2020)

7. G. Pagliai, M. Dinu, M. Bonaccio, L. Iacoviello, F. Sofi, British Journal Of Nutrition, 125(3) (2020) 
8. T. Shimizu. Nutrition Research Reviews, 16 (2003)

9. A.B. Lisitsyn, I.M. Chernukha, O.I. Lunina, Theory and practice of meat processing, 3(1) (2018)

10. M. Shimizu, Food Journal of Food and Drug Analysis, 20(1) (2012)

11. C. Georgescu, A.G. Birka, N.I. Myachikova, Research result, Technologies of business and service, 3(13) (2016)

12. I. Tomašević, P. Putnik, F. Valjak, B. Pavlić, Current Opinion in Food Science, 41 (2021)

13. E. Lee, Jang-E. Lee, Current Opinion in Food Science, 37 (2021)

14. Brasil, Ministério da Saúde (2012) http://www.brasil.gov.br/ 